Document downloaded from:

http://hdl.handle.net/10251/83353

This paper must be cited as:

Carceller Candau, C.; Soto Pacheco, P.; Boria Esbert, VE.; Guglielmi, M. (2016). Capacitive Obstacle Realizing Multiple Transmission Zeros for In-Line Rectangular Waveguide Filters. IEEE Microwave and Wireless Components Letters. 26(10):795-797. doi:10.1109/LMWC.2016.2605462.

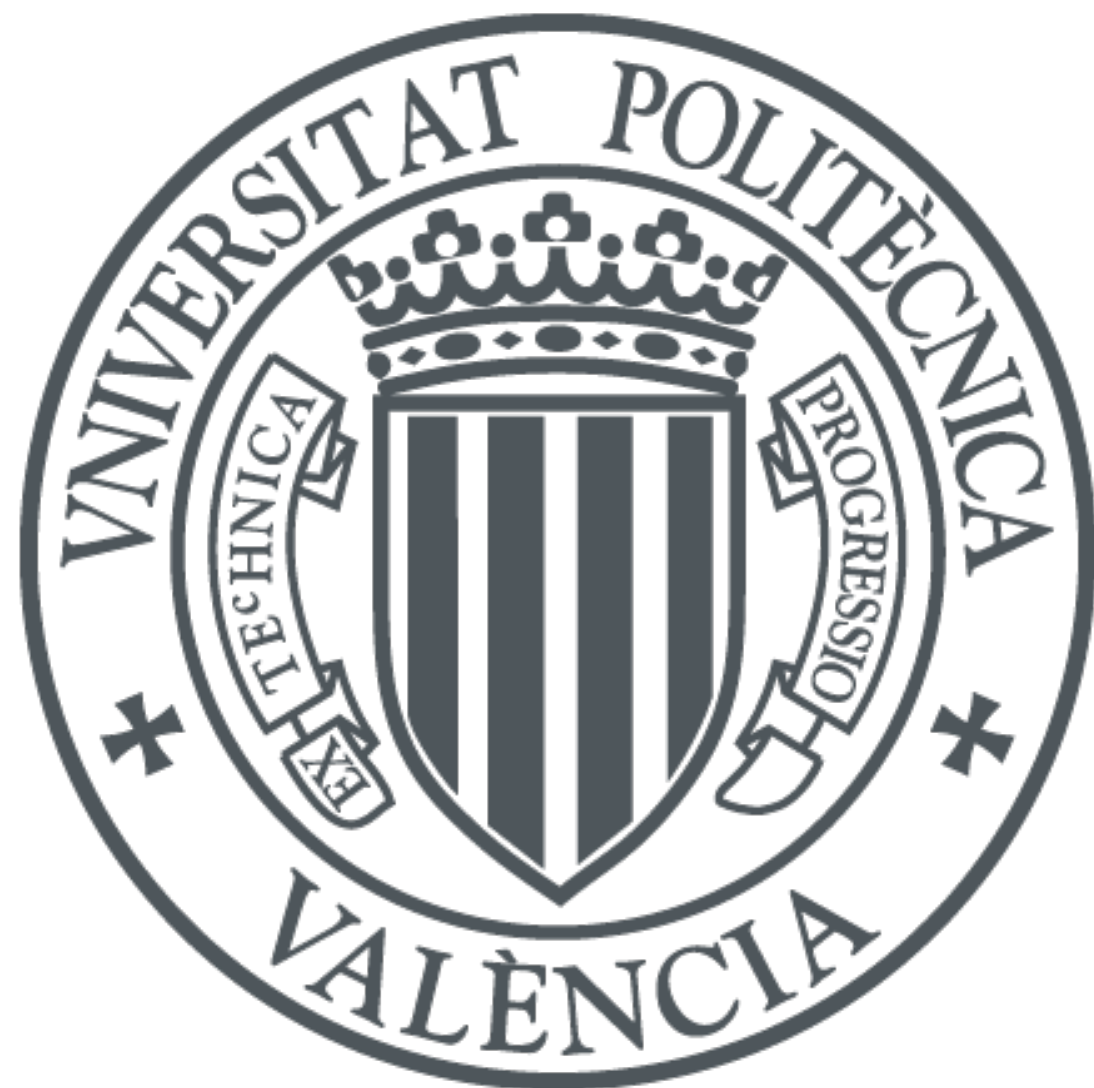

The final publication is available at

http://dx.doi.org/10.1109/LMWC.2016.2605462

Copyright Institute of Electrical and Electronics Engineers (IEEE)

Additional Information

(c) 2016 IEEE. Personal use of this material is permitted. Permission from IEEE must be obtained for all other users, including reprinting/ republishing this material for advertising or promotional purposes, creating new collective works for resale or redistribution to servers or lists, or reuse of any copyrighted components of this work in other works 


\title{
Capacitive Obstacle Realizing Multiple Transmission Zeros for In-line Rectangular Waveguide Filters
}

\author{
Carlos Carceller, Member, IEEE, Pablo Soto, Member, IEEE, Vicente Boria, Senior Member, IEEE, and \\ Marco Guglielmi, Fellow, IEEE
}

\begin{abstract}
This paper presents a capacitive obstacle for rectangular waveguide filters capable of realizing multiple transmission zeros (TZs). By combining a capacitive window with multiple thin stubs, a filter designer has the ability to control the total coupling implemented by the obstacle, and the frequency location of the TZs generated. The resulting structure is compact, and can be employed at the input/output and intermediate stages of direct-coupled-resonator filters. This letter includes a parametric analysis of the obstacle and practical design guidelines. To validate its practical application, an in-line five-pole filter realizing three TZs has been successfully realized.
\end{abstract}

Index Terms-Quasi-elliptic filters, computer-aided design, microwave filters, band-pass filters.

\section{INTRODUCTION}

$\mathbf{T}$ WO groups of techniques have been traditionally employed to generate transmission zeros (TZs) in microwave filters. One group comprises those techniques that implement multiple signal paths in the structure. This can be either done geometrically, by realizing cross-couplings between non-adjacent resonators [1], or electrically, by exciting multiple modes in certain cavities [2]. The second group of techniques uses bandstop elements to realize both transmission and in-band reflection zeros. These elements are frequently connected to the filter via non-resonating nodes (NRN) [3], in order to facilitate the design and manufacture of the structure.

In [4], an alternative approach was proposed: the usage of wide stubs to implement coupling elements in in-line Eplane filters. The purpose of each stub is twofold: generate an appropriate coupling level and also realize a TZ. This can be achieved by adjusting its height and width. Although practical in many applications, this mechanism has some drawbacks. The implementation of moderate and weak coupling levels between resonators is difficult. It often requires bulky solutions with extremely high stubs (note that the examples reported in [4] include the stubs at the input and output stages). Given the finite range of coupling levels available, the frequency band where the TZ can be realized is quite limited (usually around the passband). As reported in [4], a wider stub can introduce a pair of TZs. This property helps mitigate the limitation in

C. Carceller, P. Soto, V. Boria and M. Guglielmi are with Universitat Politecnica de Valencia, 46022, Valencia, Spain. Email: carcarc2@upvnet.upv.es, pabsopac@dcom.upv.es, vboria@dcom.upv.es, marco.guglielmi@iteam.upv.es.

This work was supported by the Spanish Ministerio de Economia y Competitividad under R\&D project TEC2013-47037-C5-1-R.

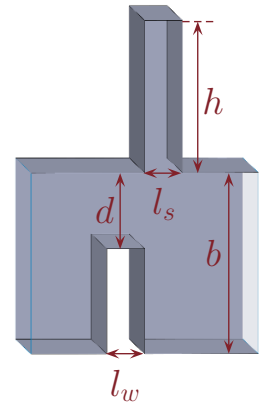

Fig. 1. Capacitive obstacle including one stub (side wall removed).

TZ tunability, but a quite longer structure is obtained, and the resulting TZs cannot be independently controlled.

In the present paper, a new capacitive obstacle is introduced, which can substitute any iris in in-line rectangular waveguide filters. It is based on the connection of up to two E-plane stubs to a classical capacitive window. The obstacle is responsible for providing an adequate coupling level between adjacent resonators while realizing one $\mathrm{TZ}$ per stub. By isolating the adjustment of the coupling level from the realization of TZs, a more compact obstacle can be implemented. Likewise, it provides greater flexibility to control both values (coupling level and TZ frequency). The range of couplings that this obstacle is able to provide is considerably larger than the one in [4], thus it can be used at the input/output stages as well as at any intermediate iris of the filter. At the same time, each TZ can be located in a wide frequency range. With this obstacle, filters with $\mathrm{N}$ resonators are able to realize up to $2 \mathrm{~N}+2 \mathrm{TZs}$.

\section{Design Methodology}

The basic building block is depicted in Fig. 1. It is formed by a capacitive window attached to one or two thin stubs (one on each side). To simplify the study, one stub is first considered, and results can be extrapolated to the two-stub case. Design parameters are two: stub length $h$ and window height $d$.

The role of the stub is to realize a TZ. Making use of the equivalent circuit of an E-plane T-junction (described in [5]) connected on its branching port to a transmission line of electrical length $\beta h$ (and normalized characteristic admittance $b / l_{s}$ ), we conclude that this structure realizes a TZ when:

$$
\beta h=n \pi+\tan ^{-1}\left[\frac{b}{l_{s}}\left(\frac{B_{b}+B_{c}}{B_{c} B_{d}+B_{b} B_{d}-B_{c} B_{b}}\right)\right]
$$




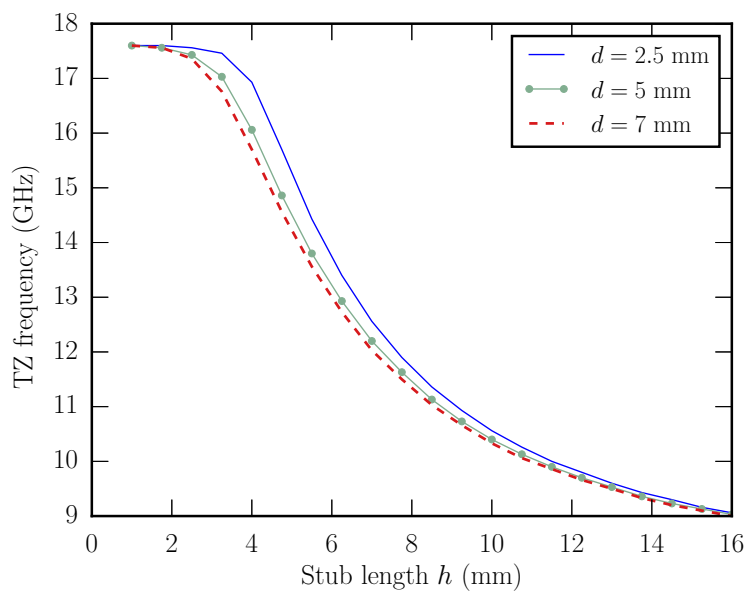

Fig. 2. TZ frequency as a function of the stub length $h$ for different values of $d$. The stub thickness $\left(l_{s}\right)$ and window length $\left(l_{w}\right)$ are set to $2 \mathrm{~mm}$.

where $B_{b}, B_{c}, B_{d}$ are reactances defined in [5] and $n$ can take any integer value. Initially, the width $l_{s}$ is fixed to a small value (normally limited by manufacturing constraints) to avoid bulky stubs. Thus, the TZ location can be controlled by adjusting $h$.

Once length $h$ yields a proper location of the TZ, the capacitive window height $d$ is adjusted until the obstacle provides an adequate normalized inverter value $\bar{K}$. It is obtained from full-wave EM simulations as:

$$
\bar{K}=\sqrt{\frac{1-\left|S_{11}\right|}{1+\left|S_{11}\right|}}
$$

The reason to adjust the $\mathrm{TZ}$ first, and then the inverter value, is that the stub dimensions have an important effect on the total coupling level. However, the effect of the window dimensions on the location of the $\mathrm{TZ}$ is much smaller. This is confirmed by EM simulations of one of these obstacle implemented in standard WR-75 waveguide. Figure 2 depicts the TZ frequency as a function of the stub length for different capacitive window sizes. As shown, the TZ can be located in a wide frequency range. At the same time, it is barely affected by a moderate change in height $d$. In contrast, Fig. 3 represents the variation of the normalized impedance inverter parameter with the height of the capacitive window. For each curve, the stub length $h$ is slightly adjusted to keep the TZ at exactly the same frequency. As expected, the normalized inverter value can be controlled by adjusting the height of the capacitive window. However, for different TZ locations, the required window height differs.

As the TZ approaches the filter center frequency, the maximum normalized inverter value that can be achieved decreases. In Fig. 3, the normalized inverter is computed at $12.6 \mathrm{GHz}$. As can be seen, the curve associated with a $\mathrm{TZ}$ at $12 \mathrm{GHz}$ has much smaller values than the other two curves, associated with TZs that are farther from the simulation frequency. Certain applications may require large coupling levels, while simultaneously ensuring that TZs are placed close to the passband. For instance, a filter with relative moderate bandwidth where the input coupling is implemented by the

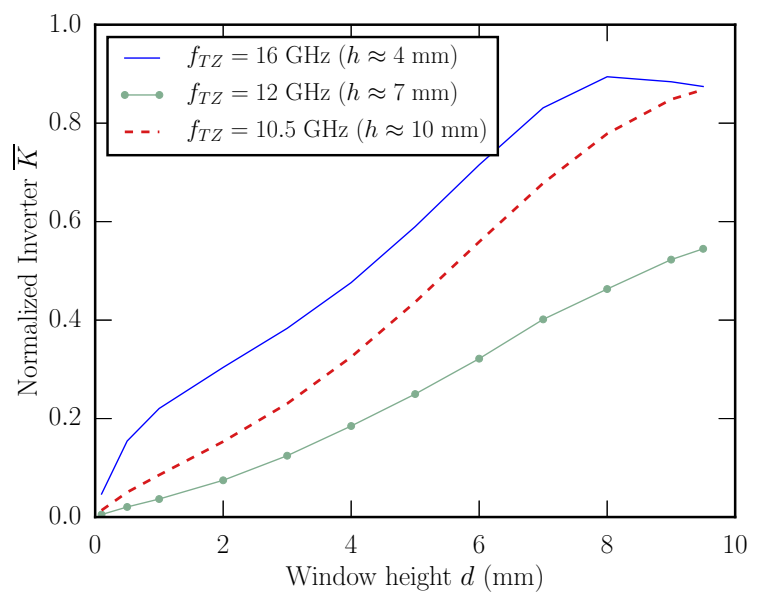

Fig. 3. Normalized impedance inverter parameter as a function of the window height $d$. Simulations are performed at $12.6 \mathrm{GHz}$. For each curve, the stub length $h$ is slightly adjusted to keep the TZ at the same frequency.

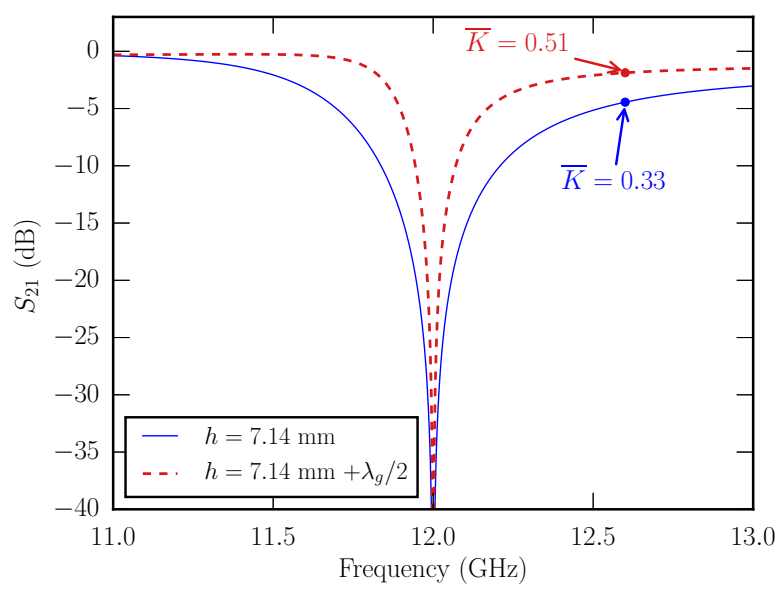

Fig. 4. Two different stub solutions, separated by $\lambda_{g} / 2$, are able to realize the same TZ but enable the implementation of different inverter values.

proposed iris-stub combination. In that scenario, we can take advantage of the fact that there are multiple solutions for the stub length that yield the same TZ location, according to (1). Take, for instance, the $S_{21}$ parameter response of Fig. 4. One curve corresponds to the simulation of a stub-iris structure with $h=7.14 \mathrm{~mm}, d=6 \mathrm{~mm}, l_{s}=l_{w}=2 \mathrm{~mm}$. The other curve corresponds to the same structure except for the stub, which is $\lambda_{g} / 2$ longer. The TZ associated with this longer stub has a much narrower bandwidth. This means that it can provide larger values of the $S_{21}$ parameter for frequencies close to the TZ. Consequently, the $S_{11}$ parameter is smaller near the TZ, and thus the normalized inverter $\bar{K}$ is larger. Effectively, by increasing the length of the stub by $\lambda_{g} / 2$ we can implement larger coupling elements while still realizing TZs that are close to the passband. However, these solutions may generate unwanted resonances in the stopband. For that reason, they are only employed if shorter solutions are not able to yield an adequate inverter value. 
Another interesting application of these obstacles is found in filters that contain very thin coupling windows, which may be difficult to manufacture. The introduction of a stub next to a capacitive window reduces the overall coupling provided by the obstacle. To compensate for the loss of coupling, the size of the capacitive window has to be increased. This is actually a positive consequence, since the window is now easier to manufacture, and definitely more robust to potential manufacturing deviations.

The main drawback of this obstacle is that it generates undesired resonances associated with the stub, limiting the spurious-free band. To shift these resonances away from the stopband, several solutions apply: readjusting the stub width $l_{s}$, changing the wall (top or bottom) where the stubs are placed and reducing the height of the adjacent resonator.

\section{RESULTS}

To demonstrate the applicability of this coupling structure to practical structures, a five-pole all-capacitive filter centered at $12.6 \mathrm{GHz}$ with a bandwidth of $400 \mathrm{MHz}$ has been designed. This structure includes three stubs, realizing TZs on both sides of the passband.

The highest-frequency TZ is adjacent to the passband, separated $170 \mathrm{MHz}$ from the edge. As was previously discussed and shown in Fig. 3, this fact limits the normalized inverter value that can be achieved by the stub-iris combination. In a Chebyshev filter, the irises that implement the smallest impedance inverters are the central ones. Consequently, the stub associated with this $\mathrm{TZ}$ is implemented there. The two TZs below the passband could be assigned to any other iris, since there seems to be no serious coupling limitation. In this case, they are both assigned to the other central iris (see Fig. 5a), demonstrating the capability of a single capacitive window to support multiple stubs.

An initial value for the normalized impedance inverters can be obtained from an all-pole distributed model [6]. From these values, the all-pole version of the filter is constructed. Then, stubs are introduced in order to generate the TZs. This forces a readjustment of the corresponding coupling windows to recover the filter response in its passband. The resulting structure is shown in Fig. 5a. In contrast with all-pole filters, note that the central coupling windows (with stubs) are not the narrowest ones, thus improving the manufacturing sensitivity and the power-handling capability of the filter.

A prototype has been manufactured in bare aluminum. Measurements are successfully compared, in Fig. 5b, with full-wave simulations provided by FEST3D. As can be seen, the location of the TZs is perfectly predicted by the EM simulations with a maximum deviation of $14 \mathrm{MHz}$. It is also clear that the presence of a TZ close to the upper edge of the passband makes the in-band response around this edge more sensitive to manufacturing deviations. However, this effect was expected after performing a tolerance analysis [7] on the filter. Therefore, this issue can be compensated in practical applications with strict passband specifications and wider design margins.

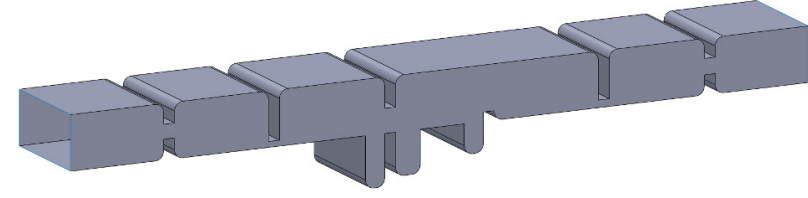

(a)

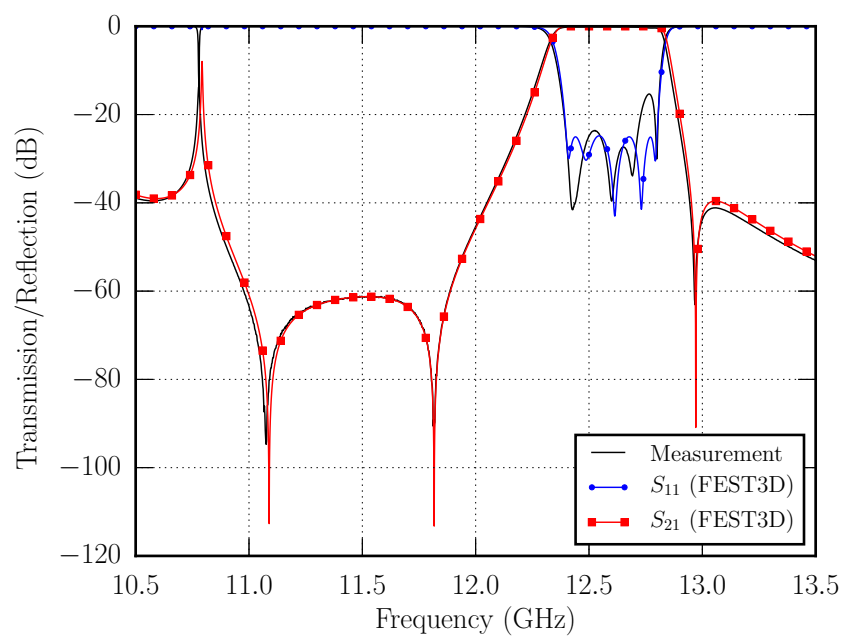

(b)

Fig. 5. a) Physical structure of the designed filter containing three stubs. b) Measurements from a manufactured prototype are successfully compared with EM simulations.

\section{CONCLUSION}

This paper proposes a new capacitive rectangular waveguide obstacle that enables the realization of TZs in a simple and direct way. This block is composed of one or two stubs attached to a classical capacitive window, thus enabling the realization of up to two TZs. This compact obstacle offers great flexibility to prescribe the location of the $\mathrm{TZ}$ in a wide frequency range, and can be employed to implement external and inter-resonator couplings. Practical guidelines for designing filters including this type of obstacle have been given. Likewise, a filter prototype has been designed and manufactured to illustrate the practical use of this obstacle.

\section{REFERENCES}

[1] R. M. Kurzrok, "General three-resonator filters in waveguide," IEEE Trans. Microw. Theory Techn., vol. 14, no. 1, pp. 46-47, Jan. 1966.

[2] A. E. Atia and A. E. Williams, "Narrow-bandpass waveguide filters," IEEE Trans. Microw. Theory Techn., vol. 20, no. 4, pp. 258-265, Apr. 1972.

[3] S. Amari and G. Macchiarella, "Synthesis of inline filters with arbitrarily placed attenuation poles by using nonresonating nodes," IEEE Trans. Microw. Theory Techn., vol. 53, no. 10, pp. 3075-3081, Oct. 2005.

[4] S. Amari and J. Bornemann, "Using frequency-dependent coupling to generate finite attenuation poles in direct-coupled resonator bandpass filters," IEEE Microw. Guided Wave Lett., vol. 9, no. 10, pp. 404-406, Oct. 1999.

[5] N. Marcuvitz, Waveguide Handbook. New York: McGraw-Hill, 1951.

[6] G. Matthaei, E. M. T. Jones, and L. Young, Microwave Filters, Impedance-Matching Networks, and Coupling Structures. New York: McGraw-Hill, 1964.

[7] P. Soto, V. E. Boria, C. Carceller, C. P. Vicente, J. Gil, and B. Gimeno, "EM-based synthesis and design of bandpass waveguide filters including manufacturing effects with FEST 3D," Int. J. RF and Microwave CAE, vol. 22, no. 1, pp. 93-103, 2012. 